\title{
All-electric detection of the polarization state of terahertz laser radiation
}

\author{
S. D. Ganichev, ${ }^{1, a)}$ W. Weber, ${ }^{1}$ J. Kiermaier, ${ }^{1}$ S. N. Danilov, ${ }^{1}$ P. Olbrich, ${ }^{1}$ D. Schuh, ${ }^{1}$ \\ W. Wegscheider, ${ }^{1}$ D. Bougeard, ${ }^{2}$ G. Abstreiter ${ }^{2}$ and W. Prettl ${ }^{1}$ \\ ${ }^{1}$ Terahertz Center, University of Regensburg, 93040, Regensburg, Germany \\ ${ }^{2}$ Walter Schottky Institute, TU Munich, Garching, Germany
}

(Received 17 March 2008; accepted 28 March 2008; published online 6 June 2008)

Two types of room-temperature detectors of terahertz laser radiation have been developed which allow, in an all-electric manner, the determination of the plane of polarization of linearly polarized radiation and the Stokes parameters of elliptically polarized radiation, respectively. The operation of the detectors is based on photogalvanic effects in semiconductor quantum well structures of low symmetry. The photogalvanic effects have nanosecond time constants at room temperature, making a high time resolution of the polarization detectors possible. (c) 2008 American Institute of Physics. [DOI: $10.1063 / 1.2937192]$

\section{INTRODUCTION}

Terahertz (THz) physics and technology are on the frontiers of physics, holding great promise for progress in various fields of science such as solid-state physics, astrophysics, plasma physics, and others (see, e.g., Refs. 1-4). Furthermore, $\mathrm{THz}$ physics presents a potential for applications in medicine, environmental monitoring, high-speed communication, security, spectroscopy of different materials, including explosives, etc. ${ }^{3-6}$ Areas of $\mathrm{THz}$ physics of high current interest are the development and application of coherent semiconductor sources, molecular gas lasers, ultrafast timedomain spectroscopy based on femtosecond near-infrared radiation pulses, as well as the development of novel detectors of laser radiation. An important characteristic of $\mathrm{THz}$ laser radiation is its state of polarization. The detection of the polarization state, in particular the orientation of the electric field vector of linear polarized radiation and/or the ellipticity of transmitted, reflected, or scattered light represents a powerful technique for analyzing the optical anisotropy of various media such as solids, solid surfaces, plasmas, and biological tissues. The established way to gain information about the polarization state is the use of optical elements, which allow the determination of optical path differences.

Here, we report on all-electric room-temperature semiconductor detector systems which provide information about the polarization state of $\mathrm{THz}$ laser radiation. The operation of detectors is based on the photogalvanic effects in semiconductor quantum well $(\mathrm{QW})$ structures of suitably low symmetry. The time constant of photogalvanic currents is determined by the momentum relaxation time of electrons, which is in the range of picoseconds at room temperature. This allows measurement of the ellipticity of $\mathrm{THz}$ laser radiation with subnanosecond time resolution. Preliminary results demonstrating the method have been published in Ref. 7 . Here, we present a detailed study of the detection principle comprising the phenomenological theory of the physical effects used for detection, the coupling of detector signals to

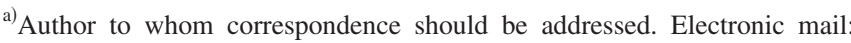
sergey.ganichev@physik.uni-regensburg.de.
}

Stokes parameters, ${ }^{8}$ the application of the method to lowintensity $\mathrm{cw}$ lasers, spectral behavior in the terahertz range, dependence on the angle of incidence, and temperature. Finally, we extend the method to the detection of the azimuth angle of linear polarized $\mathrm{THz}$ radiation.

\section{PRINCIPLE OF OPERATION}

\section{A. Photogalvanic effect}

The photogalvanic effect denotes the generation of an electric current in a homogeneous semiconductor sample by homogeneous irradiation. This is in contrast to photovoltaic effects, like those used in solar cells, where optically generated electric charges are separated by potential barriers. Homogeneity is usually realized in the terahertz range because of the weak absorption of radiation. On a macroscopic level the photogalvanic effect can be described by writing the dc current $\boldsymbol{j}$ in powers of the Fourier amplitude of the electric field of radiation $\boldsymbol{E}(\omega)$ at the frequency $\omega$. The first possibly nonvanishing term is given by $\mathrm{y}^{2,9,10}$

$$
j_{\lambda}=\sum_{\mu, \nu} \chi_{\lambda \mu \nu} E_{\mu}(\omega) E_{\nu}^{*}(\omega),
$$

where the expansion coefficient $\chi_{\lambda \mu \nu}$ is a third-rank tensor and $E_{\nu}^{*}(\omega)=E_{\nu}(-\omega)$ is the complex conjugate of $E_{\nu}(\omega)$.

The external product $E_{\mu} E_{\nu}^{*}$ can be rewritten as a sum of a symmetric and an antisymmetric product,

$$
E_{\mu} E_{\nu}^{*}=\left\{E_{\mu} E_{\nu}^{*}\right\}+\left[E_{\mu} E_{\nu}^{*}\right],
$$

with $\quad\left\{E_{\mu} E_{\nu}^{*}\right\}=\left(E_{\mu} E_{\nu}^{*}+E_{\nu} E_{\mu}^{*}\right) / 2 \quad$ and $\quad\left[E_{\mu} E_{\nu}^{*}\right]=\left(E_{\mu} E_{\nu}^{*}\right.$ $\left.-E_{\nu} E_{\mu}^{*}\right) / 2$. This decomposition corresponds to a splitting of $E_{\mu} E_{\nu}^{*}$ into its real and imaginary parts. The symmetric term is real while the antisymmetric term is purely imaginary. Due to the contraction of the tensor $\chi_{\lambda \mu \nu}$ with $E_{\mu} E_{\nu}^{*}$, the same algebraic symmetries are projected onto the last two indices of $\chi_{\lambda \mu \nu}$. The real part of $\chi_{\lambda \mu \nu}$ is symmetric in the indices $\mu \nu$, whereas the imaginary part is antisymmetric. Antisymmetric tensor index pairs can be reduced to a single pseudovector index using the Levi-Civita totally antisymmetric tensor $\delta_{\rho \mu \nu}$. Applying this simplification, we obtain for the current due to the antisymmetric part of $E_{\mu} E_{\nu}^{*}$ 


$$
\chi_{\lambda \mu \nu}\left[E_{\mu} E_{\nu}^{*}\right]=\sum_{\rho} \gamma_{\lambda \rho} i\left(\boldsymbol{E} \times \boldsymbol{E}^{*}\right)_{\rho}=\sum_{\rho} \gamma_{\lambda \rho} \hat{e}_{\rho} P_{\text {circ }} E_{0}^{2},
$$

where $\gamma_{\lambda \rho}$ is a real second-rank pseudotensor, $E_{0}=|\boldsymbol{E}|, P_{\text {circ }}$ and $\hat{e}=\boldsymbol{q} / q$ are the degree of light circular polarization (helicity) and the unit vector pointing in the direction of light propagation, respectively.

In summary, we find for the total photocurrent

$$
j_{\lambda}=\sum_{\mu, \nu} \chi_{\lambda \mu \nu}\left\{E_{\mu} E_{\nu}^{*}\right\}+\sum_{\rho} \gamma_{\lambda \rho} \hat{e}_{\rho} P_{\operatorname{circ}} E_{0}^{2},
$$

where $\chi_{\lambda \mu \nu}=\chi_{\lambda \nu \mu}$. In this equation the photogalvanic effect is decomposed into two distinct phenomena, the linear photogalvanic effect (LPGE) and the circular photogalvanic effect (CPGE), described by the first and the second term on the right-hand side, respectively. ${ }^{2,9,10}$ We note that the second term also describes the optically induced spin-galvanic effect. ${ }^{2,10-12}$ Both photogalvanic currents have been observed in various semiconductors and are theoretically well understood (for reviews see, e.g., Refs. 2 and 9-11 and references therein).

From Eq. (4), it follows that photogalvanic currents are determined by the degree of linear polarization and the orientation of the polarization ellipse as well as the handedness of elliptical polarization. In the following, we rewrite Eq. (4) for $C_{S}$ symmetry corresponding to the structure applied for detection, first for linear polarized radiation (LPGE) and afterwards for elliptically polarized radiation (both LPGE and CPGE).

\section{B. Linear polarization}

The LPGE makes it possible to determine the orientation of polarization of linearly polarized radiation. In fact, LPGE represents a microscopic ratchet. The periodically alternating electric field superimposes a directed motion on the thermal velocity distribution of carriers in spite of the fact that the oscillating field neither does exert a net force on the carriers nor induce a potential gradient. The directed motion is due to nonsymmetric random relaxation and scattering in the potential of a noncentrosymmetric medium. The linear photogalvanic effect is usually observed under linearly polarized optical excitation but may also occur under elliptically polarized radiation. It is allowed only in noncentrosymmetric media of piezoelectric crystal classes where nonzero invariant components of the third-rank tensor $\chi_{\lambda \mu \nu}$ exist. LPGE was studied in bulk crystals and has also been observed in quantum wells.

The LPGE in bulk crystals has been proposed for detection of the plane of polarization of linearly polarized radiation in Ref. 13. In bulk GaAs crystals of $T_{d}$ symmetry, irradiation with linearly polarized radiation propagating in the [111] crystallographic direction yields transverse currents along the $[1 \overline{1} 0]$ and $[11 \overline{2}]$ axes. After Eq. (4), these currents are given by

$$
j_{[1 \overline{1} 0]}=C I \times \sin 2 \alpha, \quad j_{[11 \overline{2}]}=C I \times \cos 2 \alpha,
$$

where $\alpha$ is the angle between the plane of polarization and the $[11 \overline{2}]$ axis, $I$ is the radiation intensity, and $C$ is a constant factor that both currents have in common. Equations (5) show that simultaneous measurements of the two currents allows one immediately to obtain $\alpha$, i.e., to determine the space orientation of the radiation polarization plane. A polarization analyzer made of $\mathrm{GaAs}$ crystals has been proved to give a reasonable signal from $9 \mu \mathrm{m}$ to about $400 \mu \mathrm{m}$ wavelength at room temperature.

Application of GaAs quantum well structures extends the material class suitable for detection of linear polarization. For the detection of the radiation polarization state, the most convenient geometry requires a normal incidence of radiation on the sample surface. A symmetry analysis shows that in order to obtain an LPGE photoresponse at normal incidence the symmetry of the QW structure must be as low as the point group $C_{s}$. This group contains only two elements: the identity and one mirror reflection. This can easily be obtained in QW structures by choosing a suitable crystallographic orientation. This condition is met, for instance, in (113)- or asymmetric (110)-grown structures. We introduce here a coordinate system $(x y z)$ defined by

$$
x\|[1 \overline{1} 0], \quad y\|[33 \overline{2}], \quad z \|[113],
$$

which is convenient for (113)-grown samples used here for detectors. In this coordinate system $x$ is normal to the only nonidentity symmetry element of $C_{s}$, the mirror plane.

The point group $C_{s}$ allows a LPGE current at normal incidence of the radiation on the sample because in this case the tensor $\chi$ has nonzero components $\chi_{x x y}=\chi_{x y x}, \chi_{y x x}$, and $\chi_{y y y}$. Then, after Eq. (4) the current is given by ${ }^{2}$

$$
\begin{aligned}
& j_{x}=\chi_{x x y}\left(E_{x} E_{y}^{*}+E_{y} E_{x}^{*}\right), \\
& j_{y}=\chi_{y x x}\left|E_{x}\right|^{2}+\chi_{y y y}\left|E_{y}\right|^{2},
\end{aligned}
$$

yielding for linearly polarized light

$$
\begin{aligned}
& j_{x}=\chi_{x x y} \hat{e}_{z} E_{0}^{2} \sin 2 \beta, \\
& j_{y}=\left(\chi_{+}+\chi_{-} \cos 2 \beta\right) \hat{e}_{z} E_{0}^{2},
\end{aligned}
$$

where $\chi_{ \pm}=\left(\chi_{y x x} \pm \chi_{y y y}\right) / 2$ and $\beta$ is the azimuth angle between the plane of polarization defined by the electric field vector and the $x$-coordinate. A current response due to LPGE is allowed for both the $x$ - and $y$-directions. As in the bulk detector addressed above, Eqs. (8a) and (8b) show that measuring LPGE currents simultaneously in the $x$ - and $y$-directions allows one to determine unambiguously the azimuth angle $\beta$ of linearly polarized radiation.

\section{Elliptical polarization}

While LPGE gives an experimental access to the state of linear polarization, it cannot be used to determine the ellipticity of radiation. On the other hand, the circular photogalvanic effect depends on the helicity of the radiation field and cannot be induced by linearly polarized excitation. Hence, this effect may be applied to measure the ellipticity of radiation. The CPGE occurs in gyrotropic media only, as it is mediated by a second-rank pseudotensor. On a macroscopic 


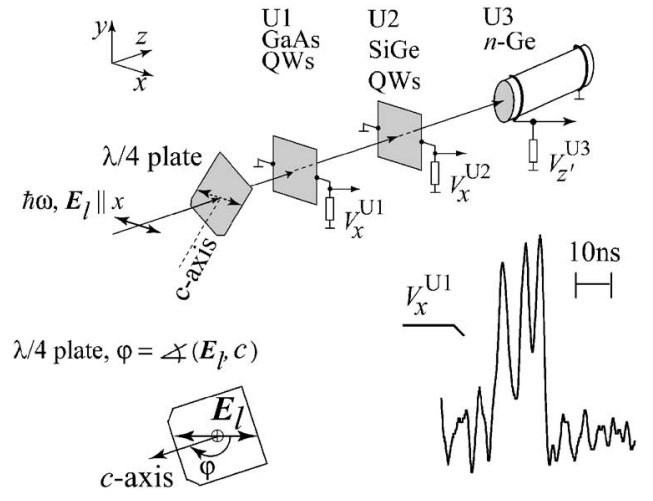

FIG. 1. Arrangement of the radiation ellipticity detector and experimental setup. The detector elements U1, U2, and U3 are GaAs QWs, a SiGe QW structure, and an $n$-Ge photon drag detector, respectively. The signal voltages $V_{x}^{\mathrm{U} 1} \propto j_{x}, V_{x}^{\mathrm{U} 2} \propto j_{x}$, and $V_{z^{\prime}}^{\mathrm{U} 3} \propto j_{z^{\prime}}$ are picked up from the elements $\mathrm{U} 1, \mathrm{U} 2$, and $\mathrm{U} 3$, across load resistors of $50 \Omega$, respectively. The ellipticity of the radiation is varied by passing linearly polarized laser radiation $\left(\boldsymbol{E}_{l} \| x\right)$ through a quarter-wave plate (bottom left). Bottom right shows the temporal structure of a typical signal pulse picked up by the element U1 after 100 times voltage amplification in a bandwidth of $300 \mathrm{MHz}$ and recorded by a broadband $(1 \mathrm{GHz})$ digital oscilloscope.

level the photocurrent of CPGE is described by the phenomenological Eq. (3) yielding $j \propto E^{2} P_{\text {circ }}$, where the radiation helicity $P_{\text {circ }}$ is given by

$$
P_{\text {circ }}=\frac{\left(\left|E_{\sigma+}\right|^{2}-\left|E_{\sigma-}\right|^{2}\right)}{\left(\left|E_{\sigma+}\right|^{2}+\left|E_{\sigma-}\right|^{2}\right)},
$$

where $\left|E_{\sigma+}\right|$ and $\left|E_{\sigma-}\right|$ are the amplitudes of right- and lefthanded circularly polarized radiation, respectively. The helicity can easily be varied by passing linearly polarized light at normal incidence through a birefringent $\lambda / 4$-plate (see Figs. 1 and 2). In this case the rotation of the quarter-wave plate by the angle $\varphi$ between the optical axis of the $\lambda / 4$-plate and the direction of the initial radiation polarization changes the

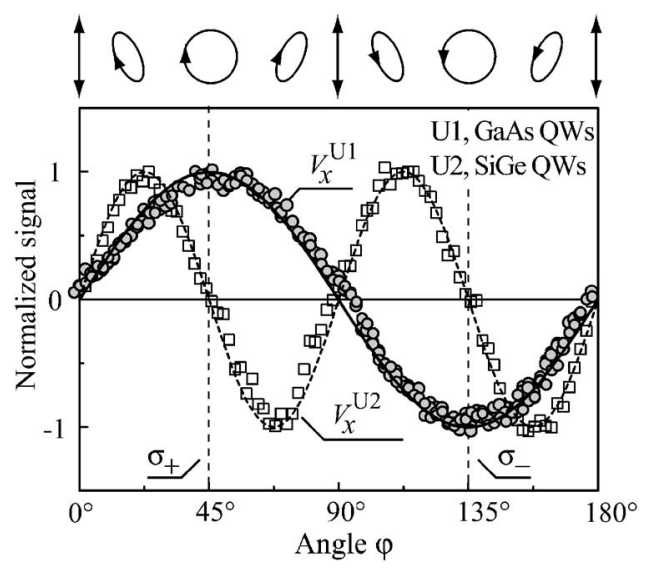

FIG. 2. Photoresponses $V_{x}^{\mathrm{U} 1}$ of the GaAs QWs (U1) and $V_{x}^{\mathrm{U} 2}$ of SiGe QWs (U2) as a function of the phase angle $\varphi$. The signals are obtained at $\lambda$ $=148 \mu \mathrm{m}$ and at room temperature. The maxima of the signal voltages are normalized to 1 . Lines are fits after $V_{x}^{\mathrm{U} 1} \propto \sin 2 \varphi$ (full line) and $V_{x}^{\mathrm{U} 2}$ $\propto \sin 4 \varphi$ (dashed line) for elements $U 1$ and $U 2$, respectively. This functional behavior agrees with the polarization dependence of the photogalvanic current given by the second and the first terms on the right-hand side of Eq. (10a), correspondingly. At the top of the figure polarization ellipses corresponding to various phase angles $\varphi$ are plotted, viewing from the direction toward which the wave is approaching. azimuth angle, the shape of the polarization ellipse, and the orientation of the rotation of the electric field vector (see Fig. 2 , top). By that $P_{\text {circ }}=\sin 2 \varphi$.

In structures of $C_{s}$ symmetry the photogalvanic current due to elliptically polarized radiation at normal incidence is after Eq. (4) given by ${ }^{2}$

$$
\begin{aligned}
& j_{x}=\chi_{x x y} \hat{e}_{z} E_{0}^{2} \sin 4 \varphi+\gamma_{x z} \hat{e}_{z} E_{0}^{2} P_{\mathrm{circ}} \sin 2 \varphi \\
& j_{y}=\left(\chi_{+}+\chi_{-} \cos ^{2} 2 \varphi\right) \hat{e}_{z} E_{0}^{2}
\end{aligned}
$$

The CPGE current is described by the second term on the right side of the first equation. It flows perpendicular to the mirror reflection plane of $C_{s}$ corresponding to the $x$-coordinate being parallel to [1 $1 \overline{1} 0]$ because the tensor $\gamma$ has a nonvanishing component $\gamma_{x z}$. The LPGE current, given by the first term on the right side Eq. (10a) and by Eq. (10b), can be generated in both the $x$ - and $y$-directions. It reflects only the projection of the electric field of the elliptical polarized radiation on the $x$ - and $y$-axes and does not contain any information about the radiation ellipticity.

Equation (10a) shows that, in general, the photogalvanic current excited by elliptically polarized radiation consists of CPGE and LPGE. In fact, the interference of both effects, CPGE and LPGE, have been observed in certain materials. ${ }^{11}$ However, CPGE and LPGE are completely independent phenomena. One or the other effect can dominate the photocurrent. As we show below, by the proper choice of materials, the information about radiation helicity can be obtained by structures with a dominating contribution of the CPGE which is proportional to the radiation helicity and carries the information about the direction of polarization vector rotation. Additional detection of a signal by a structure with dominating contribution of the LPGE provides the necessary information about azimuth angle of the polarization ellipse.

\section{Monitoring of power by photon drag effect}

For reference, it is necessary to know the power of radiation, which must be determined by a polarizationindependent sensor. For this purpose we apply the photon drag effect that is based on the transfer of linear momentum from photons to charge carriers in semiconductors. Phenomenologically, it is described by $\mathrm{by}^{2,10}$

$$
j_{l}=\sum_{m n o} T_{l m n o} q_{m} E_{n} E_{m}^{\star}
$$

where $T$ is a fourth-rank tensor and $q$ the wavevector of radiation. As a detector element we used bulk $n$-Ge of $T_{d}$ symmetry and the $\mathbf{q} \|[111]$ crystallographic direction picking up the photon drag current along the same direction (see Fig. $1)$. In this configuration, taking the coordinate $z^{\prime} \|[111]$, we get

$$
j_{z^{\prime}}=T q_{z^{\prime}}\left(\left|e_{x}^{2}\right|+\left|e_{y}^{2}\right|\right) E_{0}^{2}
$$

where 


$$
T=T_{z^{\prime} z^{\prime} x^{\prime} x^{\prime}}=T_{z^{\prime} z^{\prime} y^{\prime} y^{\prime}},
$$

in $T_{d}$ symmetry. The polarization term in Eq. (12) is equal to $1,\left|e_{x}^{2}\right|+\left|e_{y}^{2}\right|=1$; thus, the photocurrent is polarization independent.

\section{EXPERIMENTAL TECHNIQUE}

\section{A. Detector units}

To realize these detector concepts we used three detector units, U1, U2, and U3 (see Fig. 1). The U1 element is a (113)-oriented molecular beam epitaxy (MBE)-grown $p$-GaAs $/ \mathrm{Al}_{0.3} \mathrm{GaAs}_{0.7}$ multiple $\mathrm{QW}$ structure containing 20 wells of $10 \mathrm{~nm}$ width with free hole densities of about 2 $\times 10^{11} \mathrm{~cm}^{-2}$ per QW. The U2 element, also (113)-oriented, is a $\mathrm{MBE}$-grown $\mathrm{Si} / \mathrm{Si}_{0.75} \mathrm{Ge}_{0.25} / \mathrm{Si}$ single $\mathrm{QW}$ of $5 \mathrm{~nm}$ width. The SiGe QW structure is one-side boron doped with a free carrier density in the well of about $8 \times 10^{11} \mathrm{~cm}^{-2}$. For both square-shaped structures of $5 \times 5 \mathrm{~mm}^{2}$ size a pair of ohmic contacts is centered on opposite sample edges along the [1̄ㅣㄹ crystallographic axis. The unit U2 has one additional

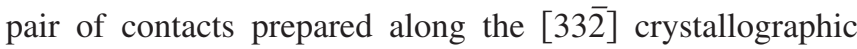
axis. As the last element (U3), we use a photon drag detector for $\mathrm{THz}$ radiation. ${ }^{2,14}$ It consists of a Ge:Sb cylinder of $5 \mathrm{~mm}$ diameter, which is about $30 \mathrm{~mm}$ long. The crystal is grown along the $z^{\prime} \|[111]$-crystallographic direction. It has plane parallel end faces and ring-shaped electric contacts at both ends (see Fig. 1). The doping level is about $10^{14} \mathrm{~cm}^{-3}$. The signal voltages are picked up independently from each detector unit in a closed-circuit configuration across a $50 \Omega$ load resistor. Signals are fed into amplifiers with voltage amplification by a factor of 100 and a bandwidth of $300 \mathrm{MHz}$ and are measured by a digital broadband (1 GHz) oscilloscope. For time-resolved measurements the signal was picked up without an amplifier.

The functionality of the polarization detectors, their sensitivity, and time resolution are demonstrated using a pulsed $\mathrm{NH}_{3} \mathrm{THz}$ laser ${ }^{2}$ with $100 \mathrm{~ns}$ pulses and a radiation power $P$ of about $10 \mathrm{~kW}$. Several lines of the $\mathrm{NH}_{3}$ laser between $\lambda$ $=76$ and $280 \mu \mathrm{m}$ have been applied. We also use a cw methanol laser with $P \approx 20 \mathrm{~mW}$ in order to check the detector applicability for detection of the $118.8 \mu \mathrm{m}$ laser line being important for tokamak applications (see, e.g., Ref. 15). The excitation of our samples at room temperature by $\mathrm{THz}$ radiation results in free carrier (Drude-type) absorption. ${ }^{2}$

To demonstrate detection of ellipticity, the polarization of the laser beam has been modified from linear to elliptical by applying $\lambda / 4$-plates made of $x$-cut crystalline quartz. The helicity of the incident light $P_{\text {circ }}=\sin 2 \varphi$ can then be varied from $P_{\text {circ }}=-1$ (left-handed circular, $\sigma_{-}$) to $P_{\text {circ }}=+1$ (righthanded circular, $\left.\sigma_{+}\right)$by changing the angle $\varphi$ between the initial linear polarization and the optical axis ( $c$-axis) of the quarter-wave quartz plate (see Fig. 1). In Fig. 2 (top) the shape of the polarization ellipse and the handedness of the radiation are shown for various angles $\varphi$.

In experiment with linear polarization, the plane of polarization has been rotated applying $\lambda / 2$ plates also made of $x$-cut crystalline quartz, which enables us to vary the azimuth angle $\beta$ between 0 and $180^{\circ}$. The rotation of the $\lambda / 2$-plate by

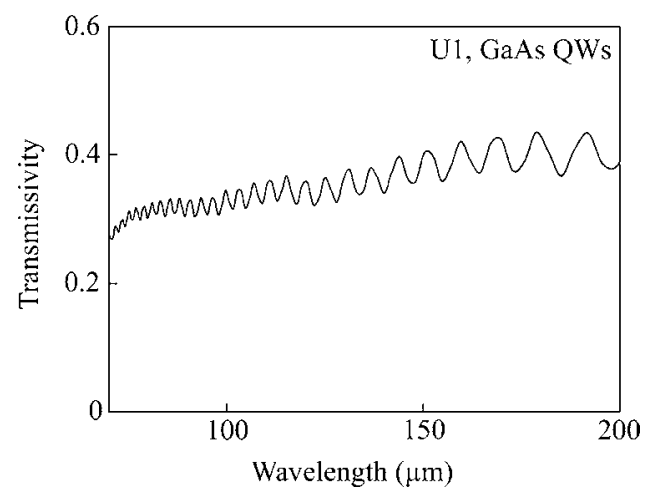

FIG. 3. Transmissivity of detector element U1 obtained by FTIR spectroscopy.

the angle $\beta / 2$ between the initial linear polarization and the optical axis of a half-wave quartz plate ( $c$-axis) leads to a rotation of the polarization plane of linearly polarized radiation by the angle $\beta$.

\section{B. Detector of elliptical polarization}

In response to irradiation of the detector $\mathrm{U} 1$ made of the GaAs QW structure, we obtain a voltage signal, $V_{x}^{\mathrm{U} 1} \propto j_{x}$, which changes its sign upon reversing the helicity. Figure 2 shows the photocurrent as a function of the phase angle $\varphi$, revealing that the signal of the detector unit U1 closely follows the radiation helicity $\left(j_{x} \propto P_{\text {circ }}=\sin 2 \varphi\right)$. In the case of the linearly polarized radiation, corresponding to $\varphi=0$ or $90^{\circ}$, the signal $V_{x}^{\mathrm{U} 1}$ vanishes.

Passing the unit $\mathrm{U} 1$ radiation hits $\mathrm{U} 2$. This is possible because $\mathrm{U} 1$ is practically transparent in the whole $\mathrm{THz}$ range. This is demonstrated by Fourier transform infrared (FTIR) measurements. Figure 3 shows the transmissivity of the unit U1 obtained in the spectral range from 70 to $200 \mu \mathrm{m}$. The transmissivity in the whole range is about $40 \%$, which just corresponds to the reflectivity of the sample made of GaAs. Periodical modulation of the spectrum is due to interference in the plane-parallel semiconductor structure. The magnitude of the reflection and interference effects can be reduced by antireflection coatings, improving the sensitivity of the detector system. Nearly the same transmission spectrum has been measured for the unit U2. Low losses in detector units allows one to stack them one behind the other for illumination with the same laser beam.

Irradiation of the second detector unit U2 made of the SiGe QW structure also results in a signal $V_{x}^{\mathrm{U} 2} \propto j_{x}$ depending on $\varphi$, which we picked up from a pair of contacts along $x$. In contrast to U1, the signal detected by U2 vanishes for circularly polarized radiation and is given by $j_{x} \propto \sin 4 \varphi$, as depicted in Fig. 2.

The observed angular dependencies, $V_{x}^{\mathrm{U} 1} \propto \sin 2 \varphi$ in the element $\mathrm{U} 1$ and $V_{x}^{\mathrm{U} 2} \propto \sin 4 \varphi$ in the element $\mathrm{U} 2$, demonstrate that the photocurrent is caused predominately by CPGE [Eq. (10a)], second term on the right-hand side) in our GaAs QWs, and by LPGE [Eq. (10a)], first term on the right-hand side) in the present $\mathrm{SiGe}$ structures. We note that the dominance of one or the other effect (CPGE or LPGE) in a material is not a matter of course. In other samples of the same 

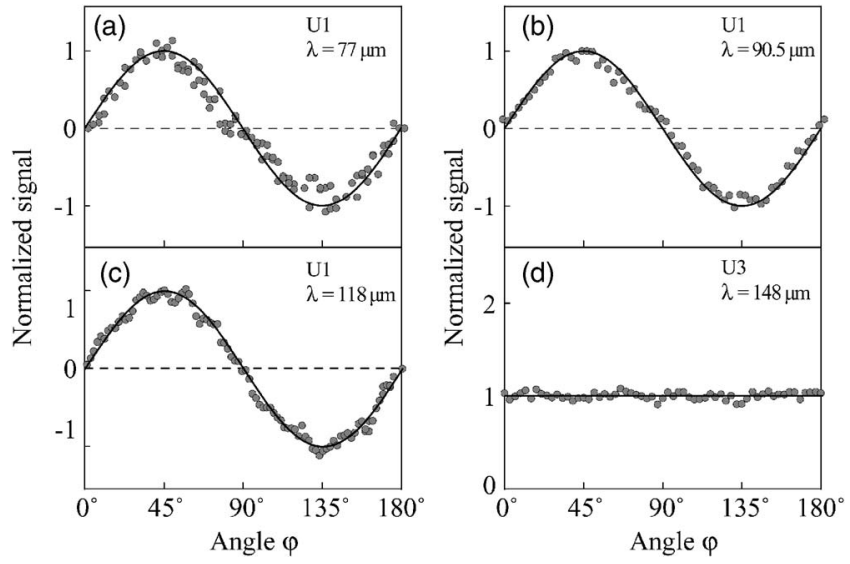

FIG. 4. Photoresponses as a function of the phase angle $\varphi$ obtained for various wavelengths. The maxima of the signal voltages are normalized to 1 . (a)-(c): photoresponse $V_{x}^{\mathrm{U} 1}$ of the GaAs QWs (U1). We emphasize that the signal plotted in (c) is obtained by a cw laser with about $20 \mathrm{~mW}$ power. Lines are fits after $V_{x}^{\mathrm{U} 1} \propto \sin 2 \varphi$. (d) Photoresponse $V_{z^{\prime}}^{\mathrm{U} 3}$ of photon drag detector element U3 demonstrating its independence of polarization state. Line is a fit after Eq. (12).

crystallographic orientation both may contribute with comparable strength, yielding a beating in the $\varphi$ dependence of the photocurrent. ${ }^{2}$ The last detector unit U3 is needed to monitor the radiation power. This unit is based on the photon drag effect, whose sensitivity in the present geometry is independent on the polarization state of radiation as shown in Fig. 4(d).

From Figs. 2 and 4(d), it follows that simultaneous measurements of all three signals allow the unambiguous determination of the radiation ellipticity and consequently the Stokes parameters. ${ }^{8}$ Indeed, a pair of signals of U1 and U2 obtained at an angle $\varphi$ is unique, and this pair of voltages will never be repeated for variation of $\varphi$ between zero and $\pi$. The ratio of the signals of $\mathrm{U} 1$ and $\mathrm{U} 2$ - and even the sign of this ratio-is different for different angles $\varphi$. The angles $\varphi$ can be easily determined from measured voltages according to

$$
\varphi=\frac{1}{2} \arccos \left(\frac{V_{x}^{\mathrm{U} 1}}{V_{x}^{\mathrm{U} 2}}\right) .
$$

Combining these measurements with the signal from U3, which yields the radiation power $V_{x}^{\mathrm{U} 3} \propto P \propto E_{0}^{2}$, we get straightforwardly the Stokes parameters which completely characterize the state of polarization of the radiation field. The Stokes parameters $s_{0}$ to $s_{3}$ are related to our measured quantities $\varphi$ and $E_{0}^{2}$ by

$$
\begin{aligned}
& s_{0}=E_{0}^{2}, \\
& s_{1}=s_{0} \frac{1+\cos 4 \varphi}{2}, \\
& s_{2}=s_{0} \frac{\sin 4 \varphi}{2},
\end{aligned}
$$

$$
s_{3}=-s_{0} \sin 2 \varphi \text {. }
$$

Sensitivities of the detector units U1, U2, and U3 at the wavelength of $148 \mu \mathrm{m}$ are $3.2 \mathrm{mV} / \mathrm{kW} \quad\left(\varphi=45^{\circ}\right)$, $1.2 \mathrm{mV} / \mathrm{kW} \quad\left(\varphi=22.5^{\circ}\right)$, and $35 \mathrm{mV} / \mathrm{kW}$, respectively. These values are obtained with $50 \Omega$ load resistors and 100 times voltage amplification for angles $\varphi$ corresponding to the maximum of the voltage signal. Figure 4 shows the $\varphi$ dependence of the signal detected by the unit U1 for three different wavelengths ranging from 77 to $118 \mu \mathrm{m}$. The figure shows that the CPGE dominates the signal in this spectral range and thus gives access to the handedness of radiation. We emphasize that the $\lambda=118 \mu \mathrm{m}$ data [Fig. 4(c)] are obtained with a cw optically pumped laser at a power of not more than $P$ $\approx 20 \mathrm{~mW}$. For this measurement we modulated our beam by a chopper with a modulation frequency of $353 \mathrm{~Hz}$ and used a low-noise preamplifier (100 times voltage amplification) and a lock-in-amplifier for signal recording. We note that in this low modulation frequency case the high time resolution of the setup is not required. Thus, we applied an open-circuit configuration connecting U1 directly to the preamplifier with an input impedance of about $100 \mathrm{M} \Omega$, which resulted in the increase of the output voltage by about 30 times. Generally, if the subnanosecond time resolution is not needed, the output voltage at a fixed radiation intensity can be substantially increased by more than one order of magnitude by increasing the load resistance. Variation of the operation temperature by $\pm 30 \mathrm{~K}$ of both $\mathrm{U} 1$ and $\mathrm{U} 2$ units did not show any considerable change of the sensitivity. As a large dynamic range is important for the detection of laser radiation, we investigated the dependence of the sensitivity of the detection system on the radiation intensity by applying $\mathrm{cw}$ and high-power pulsed radiation. We observed that the ellipticity detector remains linear up to $2 \mathrm{MW} / \mathrm{cm}^{2}$ over more than nine orders of magnitude. This is indeed not surprising because all detector units work on effects caused by Drude absorption at room temperature.

In a further experiment we checked the variation of the sensitivity due to a deviation from the normal incidence of radiation. The angle of incidence dependence of the signal for a fixed radiation helicity $\left(\varphi=45^{\circ}\right.$ for $\mathrm{U} 1$ and $\varphi=22.5^{\circ}$ for U2) are shown in Fig. 5 in comparison to calculations of photogalvanic currents in QW structures of $C_{s}$ symmetry relevant to the present experiment. As it follows from Eq. (10a), the dependence of the CPGE and LPGE currents on the angle of incidence $\theta_{0}$ is determined by the value of the projection $\hat{\boldsymbol{e}}$ on the $z$ - axis and given $\mathrm{by}^{2}$

$$
j_{x} \propto \hat{e}_{z}=t_{p} t_{s} \cos \theta
$$

where $\theta$ is the refraction angle defined by $\sin \theta=\sin \theta_{0} / n_{\omega}$. The product of the transmission coefficients $t_{p}$ and $t_{s}$ for linear $p$ and $s$ polarizations, respectively, after Fresnel's formula is given by 


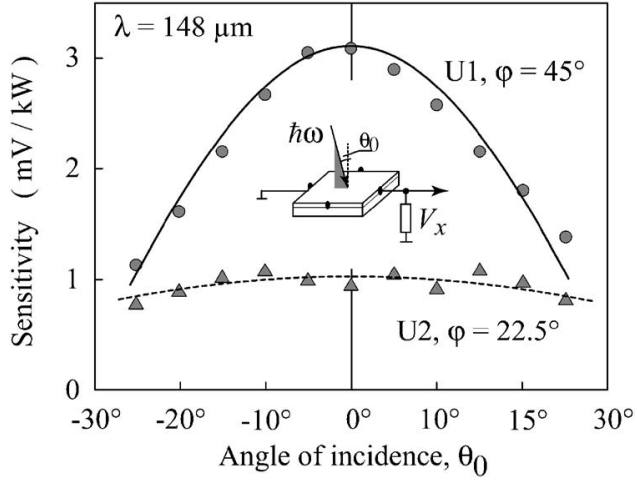

FIG. 5. Sensitivity of detector elements U1 and U2 as a function of the angle of incidence $\theta_{0}$ obtained for $\varphi=45$ and $22.5^{\circ}$, respectively. Dashed line shows the fit of the data obtained by the detector unit U2 after Eq. (15). Full line shows the fit of the data obtained by the detector unit U1 after the same Eq. (15), but taking into account an additional contribution of the circular photon drag effect given by Eq. (17).

$t_{p} t_{s}=\frac{4 n_{\omega} \cos ^{2} \theta_{0}}{\left(\cos \theta_{0}+\sqrt{n_{\omega}^{2}-\sin ^{2} \theta_{0}}\right)\left(n_{\omega}^{2} \cos \theta_{0}+\sqrt{n_{\omega}^{2}-\sin ^{2} \theta_{0}}\right)}$.

While the angle of incidence dependence of the unit U2 agrees fully with Eq. (15) (see the dashed line in Fig. 5), it does not sufficiently describe the signal of unit U1 at large angles of incidence. However, taking into account the circular photon drag effect ${ }^{16}$ in unit U1, a good agreement is obtained (see the solid line in Fig. 5). The circular photon drag effect is, like CPGE, proportional to the $P_{\text {circ }}$ and hence does not affect the basic principle of operation. Its dependence on the angle of incidence is given by ${ }^{16}$

$$
j_{x} \propto t_{p} t_{s} \sin ^{2} \theta P_{\text {circ }} .
$$

Thus, the presence of the current contribution due to the circular photon drag effect only diminishes the value of the total photocurrent at large angles of incidence. As a result we find that our whole detection system shows a tolerance of -10 to $+10^{\circ}$ for the angle of incidence.

The response time of each detector unit is due to free carrier momentum relaxation and is on the order of $10 \mathrm{ps}$ at room temperature. The real time resolution, however, is resistor-capacitor-limited by the design of the electric circuitry and by the bandwidth of the cables and amplifiers. A typical signal pulse of the unit U1 recorded by a broadband $(1 \mathrm{GHz})$ digital oscilloscope after 100 times voltage amplification in a bandwidth of $300 \mathrm{MHz}$ is shown in Fig. 1 . Additionally, we performed measurements on the unit U1 making use of the short pulse duration of the free electron laser "FELIX" at Rijnhuizen in The Netherlands. ${ }^{17}$ The FELIX operated in the spectral range between 70 and $120 \mu \mathrm{m}$. The output pulses of light from FELIX were chosen to be 3 ps long, separated by $40 \mathrm{~ns}$, in a train (or "macropulse") of $5 \mu$ s duration. The macropulses had a repetition rate of $5 \mathrm{~Hz}$. In response to 3 ps pulses, we observed that the response time of $\mathrm{U} 1$ is determined by the time resolution of our setup, but it is at least 100 ps or shorter. This fast response is typical for photogalvanics where the signal decay

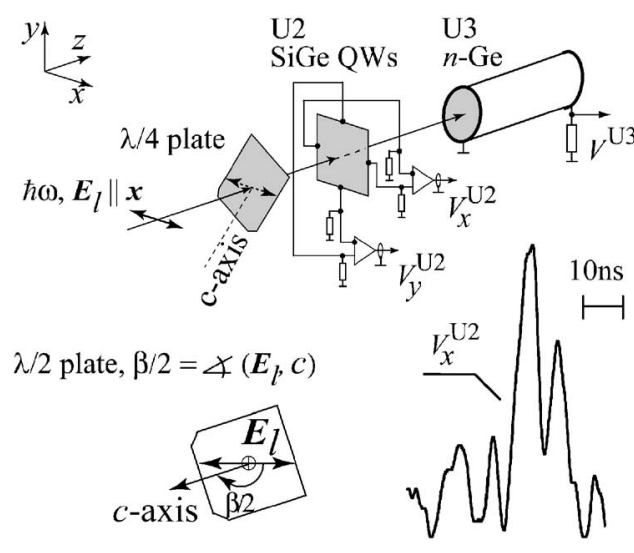

FIG. 6. Detector for the polarization of linearly polarized radiation and experimental arrangement. The detector elements U2 and U3 are a SiGe QW structure and an $n$-Ge photon drag detector, respectively. The load resistors are $50 \Omega$. The signals are fed into differential amplifiers because a common ground must be avoided and all contacts must be floating. The orientation of the plane of polarization is varied by passing linearly polarized radiation $\left(\boldsymbol{E}_{l} \| x\right)$ through a half-wave plate. The inset shows the temporal structure of a typical signal pulse of the element U2 after 100 times voltage amplification in a bandwidth of $300 \mathrm{MHz}$ and recorded by a broadband $(1 \mathrm{GHz})$ digital oscilloscope.

time is expected to be of the order of the momentum relaxation time $2,9,10$ being in our samples at room temperature of the order of $10 \mathrm{ps}$.

\section{Detector of linear polarization}

The detector of linear polarization is sketched in Fig. 6. The arrangement is indeed the same as that of the ellipticity detector (see Fig. 1), with the only difference that unit U1 is not used and at $\mathrm{U} 2$ the current signals of both contact pairs are picked up simultaneously. We note that U1 can stay in place because it is transparent.

The photocurrents $j_{x}$ and $j_{y}$ of $\mathrm{U} 2$ are measured by the voltage drops $V_{x}^{\mathrm{U} 2}$ and $V_{y}^{\mathrm{U} 2}$ across $50 \Omega$ load resistors in closed circuits. The signals are fed into differential amplifiers because a common electric ground must be avoided and all electric potentials must be floating (see Fig. 6). We note that in both amplifiers one of the inputs should be the inverted input line. These inputs are marked in Fig. 6 by dots. Figure 7 shows a measurement of both signals as a function of the polarization angle $\beta$. The full lines are fits after azimuth angle dependences given by Eqs. (8a) and (8b), demonstrating an excellent agreement to the experimental data. At the wavelength of $148 \mu \mathrm{m}$ the sensitivities of the detector unit U2 obtained with 100 times voltage amplification are 8 and $11 \mathrm{mV} / \mathrm{kW}$ for $\beta=135^{\circ}$ and $\beta=0^{\circ}$ for the signals $V_{x}^{\mathrm{U} 2}$ and $V_{y}^{\mathrm{U} 2}$, respectively. In each case the angle $\beta$ was adjusted to maximize the signal. Irradiating the detector by linear polarized light with unknown polarization angle $\beta$ yields two signals, $V_{x}^{\mathrm{U} 2}$ and $V_{y}^{\mathrm{U} 2}$, whose ratio unambiguously gives the value of the azimuth angle $\beta$. This angle is obtained by solving the equation system Eqs. (8a) and (8b). The polarization independent unit $\mathrm{U} 3$ is again used to monitor the power. We checked that its sensitivity is, as in the case of elliptical polarization, $35 \mathrm{mV} / \mathrm{kW}$ at the wavelength of $148 \mu \mathrm{m}$ and it is independent on the angle $\beta$. 


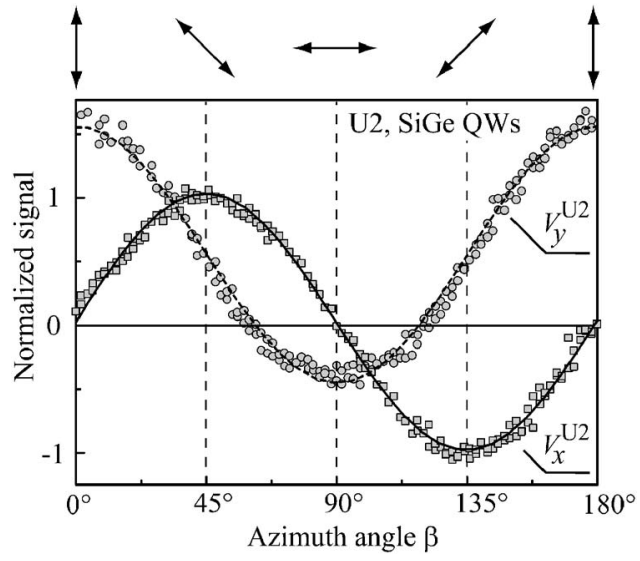

FIG. 7. Photoresponses $V_{x}^{\mathrm{U} 2}$ and $V_{y}^{\mathrm{U} 2}$ of the unit $\mathrm{U} 2$ (SiGe QWs). The signal voltages $V_{1}^{\mathrm{U} 2}$ and $V_{2}^{\mathrm{U} 2}$ are picked up by two differential amplifiers and are consequently proportional to the currents $j_{x}$ and $j_{y}$. The signals are obtained at $\lambda=148 \mu \mathrm{m}$ and at room temperature. The maxima of the signal voltages are normalized to 1 . Full and dashed lines are fits after azimuth angle dependences given by Eqs. (8a) and (8b). At the top the polarization direction corresponding to various azimuth angles $\beta$ is plotted viewing from the direction toward which the wave is approaching.

Our measurements demonstrate that all features of $\mathrm{U} 2$ in response to linear polarized radiation, such as spectral characteristic and time resolution, tolerance in angle of incidence, and dynamic range are exactly the same as those in response to elliptically polarized radiation described above. This is because in both cases the basic mechanism of response is the LPGE as given by the first term of the righthand side in Eq. (4). This term takes the form of Eqs. (8a) and $(8 b)$ for linear polarized radiation as well as the form of the first term on the right-hand side of Eqs. (10a) and (10b) for elliptically polarized radiation. In fact, the tensor $\chi$ describing all physical characteristics remains the same and only the projection of the electric radiation field on a crystallographic direction determines the polarization dependence of the current.

\section{OUTLOOK}

The sensitivities of the detector systems presented here are sufficient to detect short $\mathrm{THz}$ pulses of sources like optically pumped molecular lasers and free electron lasers. The method has also been successfully applied to monitor the ellipticity of $\mathrm{cw} \mathrm{THz}$ laser radiation by reducing the bandwidth of detection. However, we would like to point out that the sensitivity can be improved essentially by using a larger number of QWs. A further increase of sensitivity can be obtained by applying narrow-band materials such as $\mathrm{HgTe}$ QWs. Most recently we observed in HgTe QWs CPGE signals more than one order of magnitude larger than that of the GaAs QWs investigated here. ${ }^{18}$ Another way to improve sensitivity might be the application of specially designed lateral structured QWs with enhanced asymmetry. Besides other applications, sensitive $\mathrm{THz}$ detectors of the type shown here can be of particular interest for the control of current density profiles in plasmas, which is important for tokamak operation. ${ }^{19-23}$ We note that the CPGE and LPGE are also observed at valence-to-conduction band transitions, ${ }^{24-26}$ at direct intersubband transitions, ${ }^{2}$ and in wideband $\mathrm{GaN}$ semi- conductor heterojunctions. ${ }^{27}$ Thus, the applicability of the CPGE/LPGE detection scheme may be well extended into the visible, the near-infrared, and the mid-infrared spectral ranges.

\section{ACKNOWLEDGMENTS}

We thank L. E. Golub and E. L. Ivchenko for useful discussions and A. F. G. van der Meer for assistance in doing the time-resolved measurements on FELIX. The financial support from the DFG is gratefully acknowledged.

${ }^{1}$ K. Sakai, Terahertz Optoelectronics (Springer, Berlin, 2005).

${ }^{2}$ S. D. Ganichev and W. Prettl, Intense Terahertz Excitation of Semiconductors (Oxford University Press, Oxford, 2006).

${ }^{3}$ Terahertz Frequency Detection and Identification of Materials and Objects, edited by R. E. Miles, X.-C. Zhang, H. Eisele, and A. Krotkus (Springer, Berlin, 2007).

${ }^{4}$ Terahertz Science and Technology for Military and Security Applications, edited by D. L. Woolard, J. O. Jensen, and R. J. Hwu (World Scientific, Singapore, 2007).

${ }^{5}$ T. Edwards, Gigahertz and Terahertz. Technologies for Broadband Communications (Satellite Communications) (Artech House, Boston, 2000).

${ }^{6}$ Terahertz Sensing Technology: Electronic Devices and Advanced Systems Technology: 1, edited by D. L. Woolard, W. R. Loerop, and M. Shur (World Scientific, Singapore, 2003).

${ }^{7}$ S. D. Ganichev, J. Kiermaier, W. Weber, S. N. Danilov, D. Schuh, Ch. Gerl, W. Wegscheider, D. Bougeard, G. Abstreiter, and W. Prettl, Appl. Phys. Lett. 91, 091101 (2007)

${ }^{8}$ B. E. A. Saleh and M. C. Teich, Fundamentals of Photonics (John Wiley \& Sons, Inc., New York, 2007).

${ }^{9}$ B. I. Sturman and V. M. Fridkin, The Photovoltaic and Photorefractive Effects in Non-Centrosymmetric Materials (Gordon and Breach Science Publishers, New York, 1992).

${ }^{10}$ E. L. Ivchenko, Optical Spectroscopy of Semiconductor Nanostructures (Alpha Science Int, Harrow, UK, 2005).

${ }^{11}$ S. D. Ganichev and W. Prettl, J. Phys.: Condens. Matter 15, R935 (2003).

${ }^{12}$ S. D. Ganichev, E. L. Ivchenko, V. V. Bel'kov, S. A. Tarasenko, M. Sollinger, D. Weiss, W. Wegscheider, and W. Prettl, Nature (London) 417, 153 (2002) (London).

${ }^{13}$ A. V. Andrianov, E. V. Beregulin, S. D. Ganichev, K. Yu. Gloukh, and I. D. Yaroshetskii, Pis'ma Zh. Tekh. Fiz. 14, 1326 (1988) [A. V. Andrianov, E. V. Beregulin, S. D. Ganichev, K. Yu. Gloukh, and I. D. Yaroshetskii, Sov. Tech. Phys. Lett. 14, 580 (1988)].

${ }^{14}$ S. D. Ganichev, Ya. V. Terent'ev, and I. D. Yaroshetskii, Pis'ma Zh. Tekh. Fiz. 11, 46 (1985) [S. D. Ganichev, Ya. V. Terent'ev, and I. D. Yaroshetskii, Sov. Tech. Phys. Lett. 11, 20 (1985)].

${ }^{15}$ A. J. H. Donné, M. F. Graswinckel, M. Cavinato, L. Giudotti, E. Zilli, C. Gil, H. R. Koslowski, P. McCarthy, C. Nyhan, S. Prunty, M. Spillane, and C. Walker, Rev. Sci. Instrum. 75, 4694 (2004).

${ }^{16}$ V. A. Shalygin, H. Diehl, Ch. Hoffmann, S. N. Danilov, T. Herrle, S. A. Tarasenko, D. Schuh, Ch. Gerl, W. Wegscheider, W. Prettl, and S. D. Ganichev, JETP Lett. 84, 570 (2007).

${ }^{17}$ G. M. H. Knippels, X. Yan, A. M. MacLeod, W. A. Gillespie, M. Yasumoto, D. Oepts, and A. F. G. van der Meer, Phys. Rev. Lett. 83, 1578 (1999).

${ }^{18}$ B. Wittmann, S. N. Danilov, Z. D. Kvon, N. N. Mikhailov, S. A. Dvoretsky, R. Ravash, W. Prettl, and S. D. Ganichev, "Photogalvanic effects in HgTe quantum wells," in IEEE Proceedings of the Joint 32nd International Conference Infrared and Millimetre Waves and 15th International Conference THz Electronics (2007), Vol. 2, pp. 773-774; B. Wittmann, S. N. Danilov, Z. D. Kvon, N. N. Mikhailov, S. A. Dvoretsky, R. Ravash, W. Prettl, and S. D. Ganichev, e-print arXiv:cond-mat/0708.2169. ${ }^{19}$ S. A. Kazantsev, N. Ya. Polynovskaya, L. N. Pyatnitskii, and S. A. Edel'man, Usp. Fiz. Nauk 156, 3 (1988); [S. A. Kazantsev, N. Ya. Polynovskaya, L. N. Pyatnitskii, and S. A. Edel'man, Sov. Phys. Usp. 31, 785 (1988)].

${ }^{20}$ V. P. Gavrilenko, Izmer. Tekh. 35, 45 (1992); [V. P. Gavrilenko, Meas. Tech. 35, 1315 (1992)].

${ }^{21}$ F. De Marco and S. E. Segre, Plasma Phys. 14, 245 (1972).

${ }^{22}$ A. Boileau, M. von Hellermann, W. Mandl, H. P. Summers, H. Weisen, and A. Zinoviev, J. Phys. B 22, L145 (1989). 
${ }^{23}$ F. M. Levinton, R. J. Fonck, G. M. Gammel, R. Kaita, H. W. Kugel, E. T. Powell, and D. W. Roberts, Phys. Rev. Lett. 63, 2060 (1989).

${ }^{24}$ V. V. Bel'kov, S. D. Ganichev, P. Schneider, C. Back, M. Oestreich, J Rudolph, D. Hägele, L. E. Golub, W. Wegscheider, and W. Prettl, Solid State Commun. 128, 283 (2003)

${ }^{25}$ C. L. Yang, H. T. He, L. Ding, L. J. Cui, Y. P. Zeng, J. N. Wang and W. K.
Ge, Phys. Rev. Lett. 96, 186605 (2006).

${ }^{26}$ M. Bieler, N. Laman, H. M. van Driel, and A. L. Smirl, Appl. Phys. Lett. 86, 061102 (2005).

${ }^{27}$ W. Weber, S. D. Ganichev, S. N. Danilov, D. Weiss, W. Prettl, Z. D. Kvon, V. V. Bel'kov, L. E. Golub, H.-I. Cho, and J.-H. Lee, Appl. Phys. Lett. 87, 262106 (2005). 